\title{
A FINITE DIFFERENCE APPROXIMATION FOR A COUPLED SYSTEM OF NONLINEAR SIZE-STRUCTURED POPULATIONS
}

\author{
A.S. Ackleh*, H.T. Banks ${ }^{\dagger}$ and K. Deng*
}

\begin{abstract}
We study a quasilinear nonlocal hyperbolic initial-boundary value problem that models the evolution of $N$ size-structured subpopulations competing for common resources. We develop an implicit finite difference scheme to approximate the solution of this model. The convergence of this approximation to a unique bounded variation weak solution is obtained. The numerical results for a special case of this model suggest that when subpopulations are closed under reproduction, one subpopulation survives and the others go to extinction. Moreover, in the case of open reproduction, survival of more than one population is possible.
\end{abstract}

AMS subject classification. 92D25, 35A40, 65M06

\section{Introduction}

In this paper, we consider the following initial boundary value problem that describes the dynamics of coupled size-structured subpopulations with nonlinear growth, reproduction

${ }^{*}$ Department of Mathematics, University of Louisiana at Lafayette, Lafayette, Louisiana 70504.

${ }^{\dagger}$ Center for Research in Scientific Computation, North Carolina State University, Raleigh, North Carolina $27695-8205$. 
and mortality rates:

$$
\left\{\begin{array}{l}
u_{t}^{I}+\left(g^{I}(x, P(t)) u^{I}\right)_{x}+m^{I}(x, P(t)) u^{I}=0, \quad(x, t) \in\left(0, x_{\max }\right] \times(0, T], \\
g^{I}(0, P(t)) u^{I}(0, t)=C^{I}(t)+\sum_{J=1}^{N} \int_{0}^{x_{\max }} \gamma^{I, J} \beta^{J}(x, P(t)) u^{J}(x, t) d x, \quad t \in(0, T], \\
u^{I}(x, 0)=u^{I, 0}(x), \quad x \in\left[0, x_{\max }\right] .
\end{array}\right.
$$

Here $u^{I}(x, t), I=1, \ldots, N$, is the density of individuals in the $I$-th subpopulation having size $x$ at time $t$, and

$$
P(t)=\sum_{J=1}^{N} \int_{0}^{x_{\max }} w^{J}(x) u^{J}(x, t) d x
$$

is a weighted total population at time $t$. The function $m^{I}$ denotes the mortality rate of an individual in the $I$-th subpopulation, and $\beta^{I}$ is the reproduction rate of an individual in the $I$-th subpopulation. The constant parameters $0 \leq \gamma^{I, J} \leq 1$ represents the probability that an individual of the $J$-th subpopulation will reproduce an individual of the $I$-th subpopulation. The function $g^{I}$ denotes the growth rate of an individual in the $I$-th subpopulation, and $C^{I}(t)$ represents the inflow rate of the $I$-th subpopulation of zero-size individuals from an external source.

The model (1) is a generalization of several size-structured population models (often referred to as distributed rate models) which have been widely investigated in recent years (see $[8,9,15,16,18])$. Motivated by the fact that, in addition to observable characteristics such as size or age of individuals, non-observable genetic characteristics may often play a critical role in the development of the individuals, researchers in [8] presented the first such generalization of the classical Sinko-Streifer model. There, the population under consideration was treated as being composed of several subpopulations with different growth rates, i.e., there are inherent differences in growth between the individuals of the population. This results in a system of equations similar to (1) with the parameters $g^{I}, \beta^{I}$ and $m^{I}$ being independent of the total population (i.e., effect of competition is not accounted for). In [8] it 
was shown through numerical simulations that there is a crucial difference in the dynamics of the classical Sinko-Streifer models and those of the generalized models. In particular, the classical models cannot have dispersion of the density of the population in age or size. Therefore the classical models are in conflict with most of the field data collected by experimental biologists (see [8] for more details). In [9] an approximation method for the inverse problem of identifying the growth rate distribution was studied and convergence results were presented. This method was subsequently applied [18] to a semilinear model where only the mortality rate $m^{I}$ depends on the total population due to competition. Moreover, the convergence results for the inverse problem were extended to this setting. In [10] the inverse problem technique was used to fit field data (mosquitofish data which attains dispersion of the density) to the generalized linear model. The resulting data fit in [10] indicates that the need for such modification is crucial if these models were to be used as prediction tools.

When $N=1$, problem (1) reduces to a classical nonlinear Sinko-Streifer model that describes the evolution of one population with possible competition between individuals. For the linear and semilinear forms of such a model (where $g=g(x)$ and $\beta=\beta(x)$ ), several approaches have been developed in the literature for establishing existence-uniqueness of solutions. For example, in $[11,12,19]$ the semigroup of linear operators theoretic approach was used to obtain such results. Monotone approximations are developed in [1, 2], and upon passing to the limit a solution to the problem is obtained, whereas uniqueness is obtained via comparison results. For the quasilinear case (where $g=g(x, P)$ and $\beta=\beta(x, P)$ ), the well-posedness has been discussed in [3, 13], wherein completely different techniques were used for establishing the existence of a unique solution to this model. In [13] the method of characteristics together with a fixed point argument, is employed to prove this result. A difference approximation is developed in [3], and upon passing to the limit a solution to the model is obtained. Then the Holmogren Uniqueness Theorem is used to establish uniqueness of this solution. To our knowledge, results concerning existence, uniqueness, and 
convergence of approximations for the general quasilinear case given in (1) with arbitrary $N$ are not available in the literature.

In this paper, we develop an implicit finite difference approximation for problem (1). Techniques in the spirit of those in $[14,23]$ are used to obtain existence-uniqueness of weak solutions as well as convergence of the difference approximations. By a weak solution to problem (1) we mean a bounded and measurable function $u(x, t)=\left(u^{1}(x, t), u^{2}(x, t), \ldots, u^{N}(x, t)\right)$ satisfying

$$
\begin{aligned}
& \int_{0}^{x_{\max }} u^{I}(x, t) \varphi(x, t) d x-\int_{0}^{x_{\max }} u^{I, 0}(x) \varphi(x, 0) d x \\
& =\int_{0}^{t} \int_{0}^{x_{\max }}\left(u^{I} \varphi_{s}+g^{I} u^{I} \varphi_{x}-m^{I} u^{I} \varphi\right) d x d s \\
& \quad+\int_{0}^{t} \varphi(0, s)\left(C^{I}(s)+\sum_{J=1}^{N} \int_{0}^{x_{\max }} \gamma^{I, J} \beta^{J}(x, P(s)) u^{J}(x, s) d x\right) d s
\end{aligned}
$$

for $t \in[0, T], I=1, \ldots, N$, and every test function $\varphi \in C^{1}\left(\left(0, x_{\max }\right) \times(0, T)\right)$.

The following regularity conditions will be imposed on our model parameters throughout the paper: for any $I=1, \ldots, N$

(H1) $u^{I, 0}(x) \in B V\left(0, x_{\max }\right) \cap L_{\infty}\left(0, x_{\max }\right)$ and $u^{I, 0}(x) \geq 0$.

(H2) $m^{I}(x, P)$ is a nonnegative continuously differentiable function with respect to $x$ and $P$.

(H3) $\beta^{I}(x, P)$ is a nonnegative continuously differentiable function with respect to $x$ and $P$.

(H4) $g^{I}(x, P)$ is a twice continuously differentiable function with respect to $x$ and $P, g^{I}(x, P)>$ 0 for $x \in\left[0, x_{\max }\right)$, and $g^{I}\left(x_{\max }, P\right)=0$.

(H5) $C^{I}$ is a nonnegative continuously differentiable function.

(H6) $\sup _{(x, P) \in\left[0, x_{\max }\right) \times[0, \infty)} \beta^{I}(x, P) \leq \omega_{1}$. 
(H7) For any sufficiently small $\delta>0$

$$
\sup _{(x, P) \in[0, l) \times[0, \infty)}\left|\frac{g^{I}(x+\delta, P)-g^{I}(x, P)}{\delta}+m^{I}(x, P)\right| \leq \omega_{2} .
$$

(H8) $w^{I}$ is a nonnegative continuously differentiable function.

The paper is organized as follows. In Section 2, we develop a numerical scheme for computing the solution of (1) and prove the convergence of this scheme to a bounded total variation function satisfying (2). In Section 3, we present numerical results. In Section 4, we show the continuity of the weak solution under additional conditions on the initial data. Concluding remarks are given in Section 5.

\section{Convergence of Approximations}

The techniques used in this section are in the spirit of those used in $[14,23]$ to obtain convergence of finite difference approximation to conservation laws. However, it is worth pointing out that there are some major differences between problem (1) and a classical system of conservation laws. In particular, the flux in (1) is a nonlocal nonlinear function of the solution $u^{I}$ (i.e., $g^{I}=g^{I}\left(x, \sum_{J=1}^{N} \int_{0}^{x_{\max }} w^{I}(x) u^{I} d x\right)$ ), whereas it is a local nonlinear function in classical conservation laws. Furthermore, problem (1) is considered on a bounded domain $\left[0, x_{\max }\right]$ with a boundary term that is a nonlocal nonlinear function of the solution $u$, versus an unbounded domain $\mathbb{R}$ for a classical conservation law system. In the sequel, we shall show that such differences result in two problems that are very different mathematically. In particular, it is well known that for a conservation law system it is generally not possible to obtain a bound on the total variation for the approximating solutions, and hence to obtain convergence one resorts to the compensated compactness method (see, e.g., [23] for details). However, a bound for the total variation of the approximating solutions of problem (1) is established (see Lemma 3 in this section). 
The following notation will be used throughout this paper: $\Delta x=\frac{x_{\max }}{n}$ and $\Delta t=\frac{T}{m}$ denote the spatial and time mesh size, respectively. The mesh points are given by: $x_{j}=j \Delta x$, $j=0,1,2, \cdots, n$ and $t_{k}=k \Delta t, k=0,1,2, \cdots, m$. We denote by $u_{j}^{I, k}$ and $P^{k}$ the finite difference approximations of $u^{I}\left(x_{j}, t_{k}\right)$ and $P\left(t_{k}\right)$, respectively, and we let

$g_{j}^{I, k}=g^{I}\left(x_{j}, P^{k}\right), \quad \beta_{j}^{I, k}=\beta^{I}\left(x_{j}, P^{k}\right), \quad m_{j}^{I, k}=m^{I}\left(x_{j}, P^{k}\right), \quad w_{j}^{I}=w^{I}\left(x_{j}\right)$ and $C^{I, k}=C^{I}\left(t_{k}\right)$.

We define the difference operator

$$
D_{h}^{-}\left(u_{j}^{I, k}\right)=\frac{u_{j}^{I, k}-u_{j-1}^{I, k}}{\Delta x}, \quad 1 \leq j \leq n
$$

and the $l^{1}$ and $l^{\infty}$ norm of $u^{I, k}$ by

$$
\begin{aligned}
\left\|u^{I, k}\right\|_{1} & =\sum_{j=1}^{n}\left|u_{j}^{I, k}\right| \Delta x \\
\left\|u^{I, k}\right\|_{\infty} & =\max _{j=0,1,2, \cdots, n}\left|u_{j}^{I, k}\right| .
\end{aligned}
$$

We then discretize the partial differential equation in (1) using the following implicit finite difference approximation

$$
\left\{\begin{array}{l}
\frac{u_{j}^{I, k+1}-u_{j}^{I, k}}{\Delta t}+\frac{g_{j}^{I, k} u_{j}^{I, k+1}-g_{j-1}^{I, k} u_{j-1}^{I, k+1}}{\Delta x}+m_{j}^{I, k} u_{j}^{I, k+1}=0, \quad 1 \leq j \leq n \\
g_{0}^{I, k} u_{0}^{I, k+1}=C^{I, k}+\sum_{J=1}^{N} \sum_{i=1}^{n} \gamma^{I, J} \beta_{i}^{J, k} u_{i}^{J, k} \Delta x \\
P^{k+1}=\sum_{I=1}^{N} \sum_{i=1}^{n} w_{i}^{I} u_{i}^{I, k+1} \Delta x
\end{array}\right.
$$

with the initial condition

$$
u_{j}^{I, 0}=\frac{1}{\Delta x} \int_{(j-1) \Delta x}^{j \Delta x} u^{I, 0}(x) d x, \quad j=1, \cdots, n, I=1, \ldots, N .
$$

If we define

$$
d_{j}^{I, k}=1+\frac{\Delta t}{\Delta x} g_{j}^{I, k}+\Delta t m_{j}^{I, k}, \quad 1 \leq j \leq n, I=1, \ldots, N,
$$

then (3) can be equivalently written as the following system of linear equations for $\vec{u}^{k+1}=$ $\left[u_{0}^{1, k+1}, u_{1}^{1, k+1}, \ldots, u_{n}^{1, k+1}, u_{0}^{2, k+1}, u_{1}^{2, k+1}, \ldots, u_{n}^{2, k+1}, \ldots, u_{0}^{N, k+1}, u_{1}^{N, k+1}, \ldots, u_{n}^{N, k+1}\right]^{T} \in R^{N \times(n+1)}$
$A^{k} \vec{u}^{k+1}=\vec{f}^{k}$ 
where

$$
\begin{gathered}
\vec{f}^{k}=\left[C^{1, k}+\sum_{J=1}^{N} \sum_{i=1}^{n} \gamma^{1, J} \beta_{i}^{J, k} u_{i}^{J, k} \Delta x, u_{1}^{1, k}, \ldots, u_{n}^{1, k}, C^{2, k}+\sum_{J=1}^{N} \sum_{i=1}^{n} \gamma^{2, J} \beta_{i}^{J, k} u_{i}^{J, k} \Delta x\right. \\
\left.u_{1}^{2, k}, \ldots, u_{n}^{2, k}, \ldots, C^{N, k}+\sum_{J=1}^{N} \sum_{i=1}^{n} \gamma^{N, J} \beta_{i}^{J, k} u_{i}^{J, k} \Delta x, u_{1}^{N, k}, \ldots, u_{n}^{N, k}\right]^{T}
\end{gathered}
$$

and $A^{k}$ is the following block diagonal matrix

$$
A^{k}=\left(\begin{array}{ccccc}
A^{1, k} & 0 & 0 & \cdots & 0 \\
0 & A^{2, k} & 0 & \cdots & 0 \\
0 & 0 & A^{3, k} & \ldots & 0 \\
\ldots \ldots \ldots & \ldots \ldots \ldots \ldots \\
0 & 0 & 0 & 0 & A^{N, k}
\end{array}\right)
$$

with the lower triangular matrix

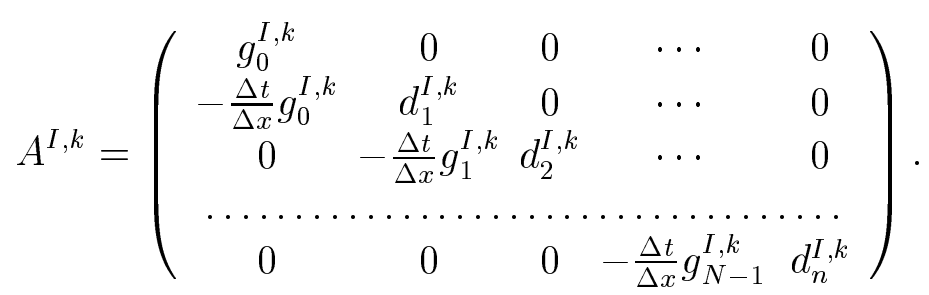

Note that using the assumptions on our parameters one can easily show that equation (4) has a unique solution satisfying $\vec{u}^{k+1} \geq 0, k=0, \ldots, m$. Next we will show that the difference approximation is bounded in $l^{1}$ norm.

Lemma 1 The following estimate holds:

$$
\sum_{I=1}^{N}\left\|u^{I, k}\right\|_{1} \leq\left(1+N \omega_{1} \Delta t\right)^{k} \sum_{I=1}^{N}\left\|u^{I, 0}\right\|_{1}+\sum_{i=1}^{k}\left(1+N \omega_{1} \Delta t\right)^{k-i} \sum_{I=1}^{N}\left|C^{I, i-1}\right| \Delta t
$$

and thus

$$
\begin{aligned}
P^{k} \leq P_{\max }= & \max _{I=1, \ldots, N}\left\|w^{I}\right\|_{\infty}\left(\left(1+N \omega_{1} \Delta t\right)^{m} \sum_{I=1}^{N}\left\|u^{I, 0}\right\|_{1}\right. \\
& \left.+\sum_{I=1}^{N} \sum_{i=1}^{m}\left(1+N \omega_{1} \Delta t\right)^{m-i}\left|C^{I, i-1}\right| \Delta t\right) .
\end{aligned}
$$


Proof. Multiply equation (3) by $\Delta x$, sum over $j=1, \cdots, n$ and $I=1, \ldots, N$ to obtain

$$
\begin{aligned}
\sum_{I=1}^{N}\left\|u^{I, k+1}\right\|_{1} & \leq \sum_{I=1}^{N}\left[\left\|u^{I, k}\right\|_{1}+\Delta t\left(C^{I, k}+\sum_{J=1}^{N} \sum_{i=1}^{n} \gamma^{I, J} \beta_{i}^{J, k} u_{i}^{J, k} \Delta x\right)\right] \\
& \leq \sum_{I=1}^{N}\left[\left\|u^{I, k}\right\|_{1}+\Delta t\left(C^{I, k}+\sum_{J=1}^{N}\left\|\beta^{J}\right\|_{\infty}\left\|u^{J, k}\right\|_{1}\right)\right] \\
& =\sum_{I=1}^{N}\left\|u^{I, k}\right\|_{1}+\sum_{I=1}^{N} \Delta t C^{I, k}+\Delta t N \sum_{J=1}^{N}\left\|\beta^{J}\right\|_{\infty}\left\|u^{J, k}\right\|_{1} \\
& \leq \sum_{I=1}^{N}\left\|u^{I, k}\right\|_{1}+\Delta t \sum_{I=1}^{N} C^{I, k}+\Delta t N \max _{I=1, \ldots, N}\left\|\beta^{I}\right\|_{\infty} \sum_{I=1}^{N}\left\|u^{I, k}\right\|_{1} .
\end{aligned}
$$

Since $\max _{I} \beta^{I}(x, P) \leq \omega_{1}$, it follows that

$$
\sum_{I=1}^{N}\left\|u^{I, k+1}\right\|_{1} \leq\left(1+N \omega_{1} \Delta t\right) \sum_{I=1}^{N}\left\|u^{I, k}\right\|_{1}+\Delta t \sum_{I=1}^{N}\left|C^{I, k}\right|,
$$

which implies the estimate.

We then establish an $l^{\infty}$ bound on the difference approximation.

Lemma 2 Assume that $\Delta t$ is chosen to satisfy $\omega_{2} \Delta t<1$. Then we have the estimate

$$
\left\|u^{I, k}\right\|_{\infty} \leq \max \left\{\left(\frac{1}{1-\omega_{2} \Delta t}\right)^{k}\left\|u^{I, 0}\right\|_{\infty}, \frac{\left\|C^{I}\right\|_{\infty}+\omega_{1} \sum_{I=1}^{N}\left\|u^{I, k-1}\right\|_{1}}{\alpha_{1}}\right\},
$$

where $\alpha_{1} \leq g^{I}(0, P), I=1, \ldots, N$.

Proof. We first note that if $\max _{i} u_{i}^{I, k+1}$ occurs at the left boundary, then from the second equation of $(3)$

$$
g_{0}^{I, k}\left|u_{0}^{I, k+1}\right| \leq\left|C^{I, k}\right|+\omega_{1} \sum_{I=1}^{N}\left\|u^{I, k}\right\|_{1} .
$$

Otherwise, suppose that for some $1 \leq j \leq n, u_{j}^{I, k+1}=\max _{i} u_{i}^{I, k+1}$. Then from the difference equation (3) we have that

$$
\left(1+\Delta t m_{j}^{I, k}+\frac{\Delta t}{\Delta x} g_{j}^{I, k}\right) u_{j}^{I, k+1}-\frac{\Delta t}{\Delta x} g_{j-1}^{I, k} u_{j-1}^{I, k+1}=u_{j}^{I, k}
$$


Rearranging terms and using the inequality $u_{j-1}^{I, k+1} \leq u_{j}^{I, k+1}$, we find

$$
\left(1+\Delta t m_{j}^{I, k}\right) u_{j}^{I, k+1}+\Delta t \frac{g_{j}^{I, k}-g_{j-1}^{I, k}}{\Delta x} u_{j}^{I, k+1} \leq u_{j}^{I, k} .
$$

Hence, by (H7) we obtain

$$
\left(1-\omega_{2} \Delta t\right) u_{j}^{I, k+1} \leq u_{j}^{I, k} \leq \max _{i} u_{i}^{I, k}
$$

which implies the estimate.

Multiplying equation (3) by $w_{j}^{I}$, summing over $j=1, \ldots, n, I=1, \ldots, N$, and using Lemmas 1-2 one can easily show that there exists a $\tilde{c}>0$ such that

$$
\left|\frac{P^{k+1}-P^{k}}{\Delta t}\right| \leq \tilde{c}
$$

The bound (5) will be used in the proof of the next lemma where we show that our approximations $u_{j}^{I, k}$ have bounded total variation. This result plays a crucial role in establishing the subsequential convergence of the difference approximation (3) to a weak solution of (1). We remark again that such a bound is not possible, in general, for a system of conservation laws (see [23]).

Lemma 3 Assume $\Delta t$ satisfies $\max \left\{\omega_{1}, \omega_{2}\right\} \Delta t<1$. Then there exists a constant $c=$ $c\left(\max _{I}\left\|u^{I, 0}\right\|_{B V}, \max _{I}\left\|C^{I}\right\|_{C^{1}(0, T)}\right)$ such that for all $k=1, \cdots, m,\left\|D_{h}^{-}\left(u^{I, k}\right)\right\|_{1} \leq c, I=$ $1, \ldots, N$.

Proof. Set $\eta_{j}^{I, k}=D_{h}^{-}\left(u_{j}^{I, k}\right)$ and apply the operator $D_{h}^{-}$to equation (3) to get

$$
\frac{\eta_{j}^{I, k+1}-\eta_{j}^{I, k}}{\Delta t}+D_{h}^{-}\left(\frac{g_{j}^{I, k} u_{j}^{I, k+1}-g_{j-1}^{I, k} u_{j-1}^{I, k+1}}{\Delta x}\right)+D_{h}^{-}\left(m_{j}^{I, k} u_{j}^{I, k+1}\right)=0, \quad 2 \leq j \leq n
$$

and for $j=1$ we have that

$$
\begin{aligned}
\frac{\eta_{1}^{I, k+1}-\eta_{1}^{I, k}}{\Delta t} & =\frac{1}{\Delta t}\left(\frac{u_{1}^{I, k+1}-u_{0}^{I, k+1}}{\Delta x}-\frac{u_{1}^{I, k}-u_{0}^{I, k}}{\Delta x}\right) \\
& =-\frac{1}{\Delta x}\left(\frac{u_{0}^{I, k+1}-u_{0}^{I, k}}{\Delta t}+D_{h}^{-}\left(g_{1}^{I, k} u_{1}^{I, k+1}\right)+m_{1}^{I, k} u_{1}^{I, k+1}\right) .
\end{aligned}
$$


Multiplying each equation by $\Delta x \operatorname{sgn}\left(\eta_{j}^{I, k+1}\right)$, using the fact that $-\eta^{I, k} \operatorname{sgn}\left(\eta_{j}^{I, k+1}\right) \geq-\left|\eta^{I, k}\right|$, and summing over the indices, $j=1,2, \cdots, n$, we find

$$
\begin{aligned}
& \frac{\left\|\eta^{I, k+1}\right\|_{1}-\left\|\eta^{I, k}\right\|_{1}}{\Delta t} \\
& \quad+\sum_{j=1}^{n}\left[D_{h}^{-}\left(\frac{g_{j}^{I, k} u_{j}^{I, k+1}-g_{j-1}^{I, k} u_{j-1}^{I, k+1}}{\Delta x}\right)+D_{h}^{-}\left(m_{j}^{I, k} u_{j}^{I, k+1}\right)\right] \operatorname{sgn}\left(\eta_{j}^{I, k+1}\right) \Delta x \leq 0,
\end{aligned}
$$

where we set $m_{0}^{I, k}=0$ and

$$
D_{h}^{-}\left(g_{0}^{I, k} u_{0}^{I, k+1}\right)=-\frac{u_{0}^{I, k+1}-u_{0}^{I, k}}{\Delta t}
$$

Now, simple calculations yield

$$
\begin{aligned}
& \sum_{j=1}^{n} D_{h}^{-}\left(\frac{g_{j}^{I, k} u_{j}^{I, k+1}-g_{j-1}^{I, k} u_{j-1}^{I, k+1}}{\Delta x}\right) \operatorname{sgn}\left(\eta_{j}^{I, k+1}\right) \Delta x \\
& \geq \sum_{j=2}^{n} D_{h}^{-}\left(\frac{g_{j}^{I, k}-g_{j-1}^{I, k}}{\Delta x} u_{j-1}^{I, k+1}\right) \operatorname{sgn}\left(\eta_{j}^{I, k+1}\right) \Delta x+\frac{u_{0}^{I, k+1}-u_{0}^{I, k}}{\Delta t} \operatorname{sgn}\left(\eta_{1}^{I, k+1}\right) \\
& \quad+D_{h}^{-}\left(g_{1}^{I, k}\right) u_{0}^{I, k+1} \operatorname{sgn}\left(\eta_{1}^{I, k+1}\right) .
\end{aligned}
$$

Thus,

$$
\begin{aligned}
& \frac{\left\|\eta^{I, k+1}\right\|_{1}-\left\|\eta^{I, k}\right\|_{1}}{\Delta t} \\
& \leq \max _{j}\left(D_{h}^{-}\left(g_{j}^{I, k}\right)+m_{j}^{I, k}\right)\left\|\eta^{I, k+1}\right\|_{1} \\
& \quad+\max _{j}\left|D_{h}^{-}\left(D_{h}^{-}\left(g_{j}^{I, k}\right)+m_{j}^{I, k}\right)\right|\left\|u^{I, k+1}\right\|_{1} \\
& \quad+\left|\frac{u_{0}^{I, k+1}-u_{0}^{I, k}}{\Delta t}\right|+\left|D_{h}^{-}\left(g_{1}^{I, k}\right)\right|\left|u_{0}^{I, k+1}\right| .
\end{aligned}
$$

From Lemmas 1-2, it suffices to obtain a bound for the term $\left|\frac{u_{0}^{I, k+1}-u_{0}^{I, k}}{\Delta t}\right|$. To this end, consider the boundary condition

$$
g_{0}^{I, k} u_{0}^{I, k+1}=C^{I, k}+\sum_{J=1}^{N} \sum_{j=1}^{n} \gamma^{I, J} \beta_{j}^{J, k} u_{j}^{J, k} \Delta x
$$


Then, using equation (3) together with summation by parts we obtain

$$
\begin{aligned}
g_{0}^{I, k} & \left(\frac{u_{0}^{I, k+1}-u_{0}^{I, k}}{\Delta t}\right)+\left(\frac{g_{0}^{I, k}-g_{0}^{I, k-1}}{\Delta t}\right) u_{0}^{I, k}-\frac{C^{I, k}-C^{I, k-1}}{\Delta t} \\
& =\sum_{J=1}^{N} \sum_{j=1}^{n} \gamma^{I, J}\left[\beta_{j}^{J, k}\left(\frac{u_{j}^{J, k}-u_{j}^{J, k-1}}{\Delta t}\right)+\left(\frac{\beta_{j}^{J, k}-\beta_{j}^{J, k-1}}{\Delta t}\right) u_{j}^{J, k-1}\right] \Delta x \\
& \leq \sum_{J=1}^{N} \sum_{j=1}^{n}\left[-\beta_{j}^{J, k}\left(D_{h}^{-}\left(g_{j}^{J, k-1} u_{j}^{J, k}\right)+m_{j}^{J, k-1} u_{j}^{J, k}\right)+\beta_{P}^{J}(x, \bar{P})\left(\frac{P^{k}-P^{k-1}}{\Delta t}\right) u_{j}^{J, k-1}\right] \Delta x \\
& \leq \sum_{J=1}^{N} \sum_{j=1}^{n}\left[\left(D_{h}^{-}\left(\beta_{j}^{J, k}\right) g_{j}^{J, k-1}-\beta_{j}^{J, k} m_{j}^{J, k-1}\right) u_{j}^{J, k}+\beta_{P}^{J}(x, \bar{P})\left(\frac{P^{k}-P^{k-1}}{\Delta t}\right) u_{j}^{J, k-1}\right] \Delta x \\
& +\sum_{J=1}^{N} \beta_{1}^{J, k} g_{0}^{J, k-1} u_{0}^{J, k},
\end{aligned}
$$

where $\bar{P}$ is between $P^{k-1}$ and $P^{k}$. Hence, using the bound given in (5) we find

$$
\begin{aligned}
& \left|g_{0}^{I, k}\left(\frac{u_{0}^{I, k+1}-u_{0}^{I, k}}{\Delta t}\right)+\left(\frac{g_{0}^{I, k}-g_{0}^{I, k-1}}{\Delta t}\right) u_{0}^{k}-\frac{C^{I, k}-C^{I, k-1}}{\Delta t}\right| \\
& \leq \sum_{J=1}^{N}\left(\sup _{j}\left|D_{h}^{-}\left(\beta_{j}^{J, k}\right) g_{j}^{J, k-1}\right|+\sup _{j}\left|\beta_{j}^{J, k} m_{j}^{J, k-1}\right|\right)\left\|u^{J, k}\right\|_{1} \\
& \quad+\sum_{J=1}^{N} \tilde{c}(x, \bar{P}) \in\left[0, x_{\max }\right] \times\left[0, P_{\max ]}\left|\beta_{P}^{J}(x, \bar{P})\right|\left\|u^{J, k-1}\right\|_{1}\right. \\
& \quad+\sum_{J=1}^{N}\left|\beta_{1}^{J, k}\right|\left(\left|C^{J, k-1}\right|+\sum_{L=1}^{N} \sup _{j}\left|\beta_{j}^{L, k-1}\right|\left\|u^{L, k-1}\right\|_{1}\right) .
\end{aligned}
$$

Now, Lemmas 1-2 imply that there exists a constant $\alpha>0$ such that $\left|\frac{u_{0}^{I, k+1}-u_{0}^{I, k}}{\Delta t}\right| \leq \alpha$. Applying this bound to (6), we conclude that there exists a constant $\omega_{3}>0$ such that

$$
\frac{\left\|\eta^{I, k+1}\right\|_{1}-\left\|\eta^{I, k}\right\|_{1}}{\Delta t} \leq \omega_{2}\left\|\eta^{k+1}\right\|_{1}+\omega_{3}
$$

and the result is established.

The next result shows that the difference approximations satisfy a Lipschitz-type condition in $t$. 
Lemma 4 Assume $\Delta t$ satisfies $\max \left\{\omega_{1}, \omega_{2}\right\} \Delta t<1$. Then there exists an $A>0$ such that for any $m>p$

$$
\sum_{j=1}^{n}\left|\frac{u_{j}^{I, m}-u_{j}^{I, p}}{\Delta t}\right| \Delta x \leq A(m-p), I=1, \ldots, N .
$$

Proof. Summing the first equation in (3) over $j$ and multiplying by $\Delta x$ we obtain

$$
\begin{aligned}
& \sum_{j=1}^{n}\left|\frac{u_{j}^{I, k+1}-u_{j}^{I, k}}{\Delta t}\right| \Delta x=\sum_{j=1}^{n}\left|-g_{j-1}^{I, k} \frac{u_{j}^{I, k+1}-u_{j-1}^{I, k+1}}{\Delta x}-u_{j}^{I, k+1} \frac{g_{j}^{I, k}-g_{j-1}^{I, k}}{\Delta x}-m_{j}^{I, k} u_{j}^{I, k+1}\right| \Delta x \\
& \quad \leq \sup _{j}\left|g_{j}^{I, k}\right| \sum_{j=1}^{n}\left|\frac{u_{j}^{I, k+1}-u_{j-1}^{I, k+1}}{\Delta x}\right| \Delta x+\sup _{j}\left|\frac{g_{j}^{I, k}-g_{j-1}^{I, k}}{\Delta x}+m_{j}^{I, k}\right|\left\|u^{I, k+1}\right\|_{1} \\
& \quad \leq \sup _{(x, P) \in\left[0, x_{\max }\right] \times\left[0, P_{\max }\right]}\left|g^{I}(x, P)\right|\left\|\eta^{I, k+1}\right\|_{1}+\omega_{2}\left\|u^{I, k+1}\right\|_{1} \leq A .
\end{aligned}
$$

Hence

$$
\sum_{j=1}^{n}\left|\frac{u_{j}^{I, m}-u_{j}^{I, p}}{\Delta t}\right| \Delta x \leq \sum_{k=p}^{m} \sum_{j=1}^{n}\left|\frac{u_{j}^{I, k+1}-u_{j}^{I, k}}{\Delta t}\right| \Delta x \leq A(m-p) . \square
$$

Following [23] we define a family of functions $\left\{U_{\Delta x, \Delta t}^{I}\right\}$ by

$U_{\Delta x, \Delta t}^{I}(x, t)=u_{j}^{I, k} \quad$ for $x \in\left[x_{j-1}, x_{j}\right), \quad t \in\left[t_{k-1}, t_{k}\right), j=1, \ldots, n, k=1, \ldots, m, I=1, \ldots, N$.

Then, the set of functions $\left\{U_{\Delta x, \Delta t}^{I}\right\}$ is compact in the topology of $L^{1}\left(\left(0, x_{\max }\right) \times(0, T)\right)$, and we have the following lemma.

Lemma 5 For $I=1, \ldots, N$ there exists a sequence $\left\{U_{\Delta x_{i}, \Delta t_{i}}^{I}\right\} \subset\left\{U_{\Delta x, \Delta t}^{I}\right\}$ which converges to a $B V\left(\left[0, x_{\max }\right] \times[0, T]\right)$ function $u^{I}(x, t)$ in the sense that for all $t>0$

$$
\int_{0}^{x_{\max }}\left|U_{\Delta x_{i}, \Delta t_{i}}^{I}(x, t)-u^{I}(x, t)\right| d x \rightarrow 0,
$$

and

$$
\int_{0}^{T} \int_{0}^{x_{\max }}\left|U_{\Delta x_{i}, \Delta t_{i}}^{I}(x, t)-u^{I}(x, t)\right| d x d t \rightarrow 0
$$


as $i \rightarrow \infty$. Furthermore, the limit function satisfies

$$
\left\|u^{I}\right\|_{B V\left(\left[0, x_{\max }\right] \times[0, T]\right)} \leq c\left(\left\|u^{I, 0}\right\|_{B V},\left\|C^{I}\right\|_{C^{1}(0, T)}\right) .
$$

Proof. The result follows from Lemmas 1-4 and the proof of Lemma 16.7 (page 276) in [23].

The next theorem will show that the limit function, $u=\left(u^{1}, u^{2}, \ldots, u^{N}\right)$, constructed via our difference scheme is actually a weak solution of problem (1).

Theorem 6 Any limit $u(x, t)=\left(u^{1}(x, t), u^{2}(x, t), \ldots, u^{N}(x, t)\right)$ defined in Lemma 5 is a weak solution of (1) and satisfies

$$
\begin{aligned}
P(t) & \leq \max _{I=1, \ldots, N}\left\|w^{I}\right\|_{\infty} \sum_{I=1}^{N}\left\|u^{I}(t)\right\|_{1} \\
& \leq \max _{I=1, \ldots, N}\left\|w^{I}\right\|_{\infty}\left(e^{\omega_{1} N T} \sum_{I=1}^{N}\left\|u^{I, 0}\right\|_{1}+\sum_{I=1}^{N} \int_{0}^{T} e^{\omega_{1}(T-s)} C^{I}(s) d s\right)=\bar{P}
\end{aligned}
$$

and

$$
\left\|u^{I}\right\|_{L^{\infty}\left(\left(0, x_{\max }\right) \times(0, T)\right)} \leq \max \left\{e^{\omega_{2} T}\left\|u^{I, 0}\right\|_{\infty}, \frac{\left\|C^{I}\right\|_{\infty}+\omega_{1} \sum_{I=1}^{N}\left\|u^{I}(t)\right\|_{1}}{\alpha_{1}}\right\},
$$

where $\alpha_{1} \leq g^{I}(0, P)$ for $P \in[0, \bar{P}], I=1, \ldots, N$.

Proof. This result can be easily established by using similar techniques as in the proof of Lemma 16.10 (page 279) in [23].

The following theorem guarantees the continuous dependence of the solution $\left\{u_{j}^{I, k}\right\}$ to (3) with respect to the initial condition $u^{I, 0}$.

Theorem 7 Let $\left\{u_{j}^{I, k}\right\}$ and $\left\{\hat{u}_{j}^{I, k}\right\}$ be the solutions of (3) corresponding to the initial conditions $u_{j}^{I, 0}$ and $\hat{u}_{j}^{I, 0}$, respectively. Then there exists a

$$
\sigma=\left(\max _{k, I}\left\|\hat{u}^{I, k}\right\|_{1}, \max _{k, I}\left\|\hat{u}^{I, k}\right\|_{\infty}, \max _{k, I}\left\|D_{h}^{-}\left(\hat{u}^{I, k}\right)\right\|_{1}\right)
$$


such that

$$
\sum_{I=1}^{N}\left\|u^{I, k+1}-\hat{u}^{I, k+1}\right\|_{1} \leq\left[1+\left(\omega_{1}+\sigma \max _{I}\left\|w^{I}\right\|\right) \Delta t\right] \sum_{I=1}^{N}\left\|u^{I, k}-\hat{u}^{I, k}\right\|_{1}
$$

for all $k \geq 0$.

Proof. Let $v_{j}^{I, k}=u_{j}^{I, k}-\hat{u}_{j}^{I, k}$ for $0 \leq k \leq m$ and $0 \leq j \leq n$. Then $v_{j}^{I, k}$ satisfies the following

$$
\left\{\begin{aligned}
\frac{v_{j}^{I, k+1}-v_{j}^{I, k}}{\Delta t}+D_{h}^{-}\left(g_{j}^{I, k} u_{j}^{I, k+1}-\hat{g}_{j}^{I, k} \hat{u}_{j}^{I, k+1}\right) & \\
+m_{j}^{I, k} v_{j}^{I, k+1}+ & \left(m_{j}^{I, k}-\hat{m}_{j}^{I, k}\right) \hat{u}_{j}^{I, k+1}=0, \quad 1 \leq j \leq n \\
g_{0}^{I, k} u_{0}^{I, k+1}-\hat{g}_{0}^{I, k} \hat{u}_{0}^{I, k+1}= & \sum_{J=1}^{N} \sum_{i=1}^{n} \gamma^{I, J} \beta_{i}^{J, k} v_{i}^{J, k} \Delta x \\
& +\sum_{J=1}^{N} \sum_{i=1}^{n} \gamma^{I, J}\left(\beta_{i}^{J, k}-\hat{\beta}_{i}^{J, k}\right) \hat{u}_{i}^{J, k} \Delta x,
\end{aligned}\right.
$$

where $\hat{g}_{j}^{I, k}=g^{I}\left(x_{j}, \hat{P}^{k}\right)$, and similar notation is used for the rest of the parameters. Multiplying each equation of $(7)$ by $\Delta x \operatorname{sgn}\left(v_{j}^{I, k+1}\right)$, summing over $j=1, \ldots, n, I=1, \ldots, N$, and using the following fact:

$$
\begin{gathered}
\sum_{j=1}^{n} D_{h}^{-}\left(g_{j}^{I, k} u_{j}^{I, k+1}-\hat{g}_{j}^{I, k} \hat{u}_{j}^{I, k+1}\right) \operatorname{sgn}\left(v_{j}^{I, k+1}\right) \Delta x \geq-g_{0}^{I, k}\left|v_{0}^{I, k+1}\right| \\
+\sum_{j=1}^{n} D_{h}^{-}\left[\left(g_{j}^{I, k}-\hat{g}_{j}^{I, k}\right) \hat{u}_{j}^{I, k+1}\right] \operatorname{sgn}\left(v_{j}^{I, k+1}\right) \Delta x,
\end{gathered}
$$

we get that

$$
\begin{aligned}
\sum_{I=1}^{N} & \frac{\left\|v^{I, k+1}\right\|_{1}-\left\|v^{I, k}\right\|_{1}}{\Delta t} \leq-\sum_{I=1}^{N} \sum_{j=1}^{n} D_{h}^{-}\left[\left(g_{j}^{I, k}-\hat{g}_{j}^{I, k}\right) \hat{u}_{j}^{I, k+1}\right] \operatorname{sgn}\left(v_{j}^{I, k+1}\right) \Delta x \\
& +\sum_{I=1}^{N} g_{0}^{I, k}\left|v_{0}^{I, k+1}\right|-\sum_{I=1}^{N} \sum_{j=1}^{n}\left(m_{j}^{I, k}-\hat{m}_{j}^{I, k}\right) \hat{u}_{j}^{I, k+1} \operatorname{sgn}\left(v_{j}^{I, k+1}\right) \Delta x \\
& -\sum_{I=1}^{N} \sum_{j=1}^{n} m_{j}^{I, k}\left|v_{j}^{I, k+1}\right| \Delta x .
\end{aligned}
$$

Now, using assumption (H4) we can easily obtain the following

$$
\begin{aligned}
-\sum_{I=1}^{N} \sum_{j=1}^{n} D_{h}^{-}\left[\left(g_{j}^{I, k}-\hat{g}_{j}^{I, k}\right) \hat{u}_{j}^{I, k+1}\right] \operatorname{sgn}\left(v_{j}^{I, k+1}\right) \Delta x & \\
& \leq\left(c_{1} \max _{I}\left\|\hat{u}^{I, k+1}\right\|_{1}+c_{2} \max _{I}\left\|\hat{u}^{I, k+1}\right\|_{\infty}+c_{3} \max _{I}\left\|D_{h}^{-}\left(\hat{u}^{I, k+1}\right)\right\|_{1}\right) \mid P^{k}-\hat{P}^{k} .
\end{aligned}
$$


Furthermore, it follows from the second equation of (3) and assumptions (H2), (H3) and (H6) that

$$
\begin{aligned}
& \sum_{I=1}^{N} g_{0}^{I, k}\left|v_{0}^{I, k+1}\right|-\sum_{I=1}^{N} \sum_{j=1}^{n}\left(m_{j}^{I, k}-\hat{m}_{j}^{I, k}\right) \hat{u}_{j}^{I, k+1} \operatorname{sgn}\left(v_{j}^{I, k+1}\right) \Delta x \\
& \quad-\sum_{I=1}^{N} \sum_{j=1}^{n} m_{j}^{I, k}\left|v_{j}^{I, k+1}\right| \Delta x \leq \omega_{1} \sum_{I=1}^{N}\left\|v^{I, k}\right\|_{1} \\
& \quad+\left(c_{4} \max _{I}\left\|\hat{u}^{I, k+1}\right\|_{1}+c_{5} \max _{I}\left\|\hat{u}^{I, k+1}\right\|_{\infty}\right)\left|P^{k}-\hat{P}^{k}\right| .
\end{aligned}
$$

Hence, choosing $\sigma>0$ so that

$$
\left(c_{1}+c_{4}\right) \max _{I}\left\|\hat{u}^{k+1}\right\|_{1}+\left(c_{2}+c_{5}\right) \max _{I}\left\|\hat{u}^{k+1}\right\|_{1}+c_{3} \max _{I}\left\|D_{h}^{-}\left(\hat{u}^{k+1}\right)\right\|_{1} \leq \sigma,
$$

we obtain

$$
\sum_{I=1}^{N}\left\|v^{I, k+1}\right\|_{1} \leq\left[1+\left(\omega_{1}+\sigma \max _{I}\left\|w^{I}\right\|_{\infty}\right) \Delta t\right] \sum_{I=1}^{N}\left\|v^{I, k}\right\|_{1},
$$

which implies the theorem.

Next, we prove that the $B V$ solution defined in Lemma 5 and Theorem 6 is unique. To this end, assume that $P(t) \in C^{1}(0, T)$ and $B^{I}(t) \in C(0, T)$ are given functions and consider the following initial-boundary value problem:

$$
\left\{\begin{array}{l}
u_{t}^{I}+\left(g^{I}(x, P(t)) u^{I}\right)_{x}+m^{I}(x, P(t)) u^{I}=0, \quad(x, t) \in\left(0, x_{\max }\right] \times(0, T], \\
g^{I}(0, P(t)) u^{I}(0, t)=B^{I}(t), \quad t \in(0, T], \\
u^{I}(x, 0)=u^{I, 0}(x), \quad x \in\left[0, x_{\max }\right] .
\end{array}\right.
$$

Then one can easily show that (8) has a unique weak solution (note that this system is uncoupled and has a local boundary condition). In fact, a weak solution can be defined as a limit of the finite difference approximation (3) with the given numbers $P^{k}=P\left(t_{k}\right)$ and uniqueness can be established by using a similar technique as in [23, Page 282]. Hence, the finite difference solution to (3) with given numbers $P^{k}=P\left(t_{k}\right)$ and $B^{I, k}=B^{I}\left(t_{k}\right)$ converges 
to the unique solution of (1) with the given $P \in C^{1}(0, T)$ and $B^{I} \in C(0, T)$. In addition, from the proof of Theorem 7 we can easily show that if $\left\{u^{k}\right\}$ and $\left\{\hat{u}^{k}\right\}$ are the solutions to (3) corresponding to given functions $\left(P^{k}, B^{I, k}\right)$ and $\left(\hat{P}^{k}, \hat{B}^{I, k}\right)$, respectively, then we have

$$
\sum_{I=1}^{N}\left\|v^{I, k+1}\right\|_{1} \leq \sum_{I=1}^{N}\left\|v^{I, k}\right\|_{1}+\sigma \Delta t\left|P^{k}-\hat{P}^{k}\right|+\Delta t \sum_{I=1}^{N}\left|B^{I, k}-\hat{B}^{I, k}\right|,
$$

where $v^{I, k}=u^{I, k}-\hat{u}^{I, k}$. Equivalently,

$$
\sum_{I=1}^{N}\left\|v^{I, k}\right\|_{1} \leq \sum_{I=1}^{N}\left\|v^{I, 0}\right\|_{1}+\sum_{i=0}^{k-1}\left[\sigma\left|P^{i}-\hat{P}^{i}\right|+\sum_{I=1}^{N}\left|B^{I, i}-\hat{B}^{I, i}\right|\right] \Delta t .
$$

Now, since from Theorem 6 for $I=1, \ldots, N,\left\{U_{\Delta x, \Delta t}^{I}\right\}$ converges to $u^{I}(x, t)$ and $\left\{\hat{U}_{\Delta x, \Delta t}^{I}\right\}$ converges to $\hat{u}^{I}(x, t)$ strongly in $C\left([0, T] ; L^{1}\left(0, x_{\max }\right)\right)$, taking the limit in (9) we obtain

$$
\sum_{I=1}^{N}\left\|v^{I}(t)\right\|_{1} \leq \sum_{I=1}^{N}\left\|v^{I}(0)\right\|_{1}+\int_{0}^{t}\left[\sigma|P(s)-\hat{P}(s)|+\sum_{I=1}^{N}\left|B^{I}(s)-\hat{B}^{I}(s)\right|\right] d s
$$

where $u(x, t), \hat{u}(x, t)$ are the unique solutions to $(8)$ given $\left(P(t), B^{I}(t)\right)$ and $\left(\hat{P}(t), \hat{B}^{I}(t)\right)$, respectively, and $v^{I}(t)=u^{I}(\cdot, t)-\hat{u}^{I}(\cdot, t)$. Then, applying the estimate given in (10) for the corresponding solutions to (8) where

$$
\begin{aligned}
P(t) & =\sum_{I=1}^{N} \int_{0}^{x_{\max }} w^{I}(x) u^{I}(x, t) d x \\
B^{I}(t) & =C^{I}(t)+\sum_{J=1}^{N} \int_{0}^{x_{\max }} \gamma^{I, J} \beta^{J}(x, P(t)) u^{J}(x, t) d x, \\
\hat{P}(t) & =\sum_{I=1}^{N} \int_{0}^{x_{\max }} w^{I}(x) \hat{u}^{I}(x, t) d x \\
\hat{B}^{I}(t) & =C^{I}(t)+\sum_{J=1}^{N} \int_{0}^{x_{\max }} \gamma^{I, J} \beta^{J}(x, \hat{P}(t)) \hat{u}^{J}(x, t) d x
\end{aligned}
$$

are defined in Theorem 6 , we obtain the following result.

Theorem 8 Suppose that $u$ and $\hat{u}$ are two bounded variation weak solutions of (1) corresponding to initial conditions $u^{0}$ and $\hat{u}^{0}$, respectively. Then

$$
\sum_{I=1}^{N}\left\|u^{I}(t)-\hat{u}^{I}(t)\right\|_{1} \leq e^{\left[\left(\sigma+N \max _{I}\left\|\beta_{P} \hat{u}\right\|_{\infty}\right) \max _{I}\left\|w^{I}\right\|_{\infty}+N \omega_{1}\right] t} \sum_{I=1}^{N}\left\|u^{I}(0)-\hat{u}^{I}(0)\right\|_{1},
$$


which means that the bounded variation weak solution to (1) is unique.

Hence, from Theorem 8 it follows that the finite difference solution converges to the unique bounded variation solution of (1).

\section{Numerical Results}

In this section, we provide some numerical results that corroborate the convergence theory presented in Section 2 and demonstrate the effect of the probability function $\gamma^{I, J}$ on the dynamics of this system. For the rest of this section we assume that $x_{\max }=1, N=2$, and the weight functions $w^{I}=1$. This implies that $P=\int_{0}^{1}\left[u^{1}(x, t)+u^{2}(x, t)\right] d x$. We choose the parameters $g^{I}, \beta^{I}, m^{I}$, and $C^{I}$ as follows:

$g^{I}(x, P)=g_{0}^{I} k^{I} f^{I}(P)(1-x), \beta^{I}(x, P)=\beta_{0}^{I}\left(1-k^{I}\right) f^{I}(P) x, m^{I}(x, P)=m^{I}(P), C^{I}(t)=0$,

where $k^{I} \in(0,1), I=1,2$. Hence, our model reduces to

$$
\left\{\begin{array}{l}
u_{t}^{I}+g_{0}^{I} k^{I} f^{I}(P)\left((1-x) u^{I}\right)_{x}+m^{I}(P) u^{I}=0, \quad x \in(0,1], t>0, \\
g_{0}^{I} k^{I} u^{I}(0, t)=\sum_{J=1}^{2} \gamma^{I, J} \beta_{0}^{J}\left(1-k^{J}\right) \int_{0}^{1} x u^{J}(x, t) d x, \quad t>0, \\
u^{I}(x, 0)=u^{I, 0}(x), \quad x \in[0,1], I=1,2 .
\end{array}\right.
$$

Integrating (11) and multiplying (11) by $x$ and integrating once again, we readily obtain the following system of differential equations:

$$
\begin{cases}\left(P^{I}\right)^{\prime}=\sum_{J=1}^{2} \gamma^{I, J} \beta_{0}^{J}\left(1-k^{J}\right) f^{J}(P) Q^{J}-m^{I}(P) P^{I} & I=1,2, \\ \left(Q^{I}\right)^{\prime}=g_{0}^{I} k^{I} f^{I}(P)\left(P^{I}-Q^{I}\right)-m^{I}(P) Q^{I}, & I=1,2,\end{cases}
$$

where $P^{I}=\int_{0}^{1} u^{I}(x, t) d x$ and $Q^{I}=\int_{0}^{1} x u^{I}(x, t) d x, I=1,2$. In our numerical simulations we have used $f^{1}(P)=e^{-0.5 P}, f^{2}(P)=e^{-0.1 P}, m^{1}(P)=0.3 P, m^{2}(P)=2 P /(1+P), k^{1}=0.5$, $k^{2}=0.7, \beta_{0}^{I}=1, g_{0}^{I}=1, t \in[0,10]$ and

$$
u^{1,0}(x)=\left\{\begin{array}{ll}
5 & x \in[0,0.3] \\
0 & x \in(0.3,1]
\end{array}, \quad u^{2,0}(x)=\left\{\begin{array}{ll}
8 & x \in[0,0.2] \\
0 & x \in(0.2,1]
\end{array} .\right.\right.
$$

To test our code we compute $\left(P^{I}, Q^{I}\right), I=1,2$, the solution of (12) using a $4-5^{\text {th }}$ order Runge Kutta routine available in MATLAB 5.3. We then compare the total population, 
$\left(P^{I}\right)^{\Delta x}(t)$, and the total biomass, $\left(Q^{I}\right)^{\Delta x}(t)$, that result from the finite difference system described in Section 2 (with $\Delta x=0.02$, and $\Delta t=0.01$ ) to the numerical solution $\left(P^{I}, Q^{I}\right)$ of the differential equation system (12) that results from the Runge Kutta routine. In Figures 1-2 we present the differences $\left(P^{I}\right)^{\Delta x}(t)-P^{I}(t)$ and $\left(Q^{I}\right)^{\Delta x}(t)-Q^{I}(t)$, respectively. The figures indicate that the finite difference approximation provides a good approximation to the solution of (11). Next we present two different dynamics for the total population and total mass depending on the choice of $\gamma^{I, J}$.

\subsection{Closed reproduction case}

In this case, we assume that reproduction is closed under subpopulations, that is, individuals in the $I$-th subpopulation only produce individuals in the $I$-th subpopulation. Hence, $\gamma^{I, I}=1, I=1,2$, and $\gamma^{I, J}=0, I \neq J$. Solving (11) we present the total populations $\left(P^{I}\right)^{\Delta x}(t), I=1,2$, and total mass $\left(Q^{I}\right)^{\Delta x}(t), I=1,2$, in Figures $3-4$, respectively. Note that in this case the second subpopulation goes to extinction. Similar phenomena have been known to occur in different structured and non-structured population models, where the surviving subpopulation is often referred to as the fittest among the others (e.g., see $[4,17])$.

\subsection{Open reproduction case}

In this case, we assume that reproduction is open under subpopulations, that is, a subpopulation of type $I$ also reproduces individuals of type $J$. For our numerical example we set $\gamma^{I, J}=\frac{1}{2}, I, J=1,2$. However, we have performed many other numerical experiments with different positive $\gamma^{I, J}$, and the dynamics are essentially the same. In Figures 5-6 we present the total subpopulations $\left(P^{I}\right)^{\Delta x}(t), I=1,2$, and total mass $\left(Q^{I}\right)^{\Delta x}(t)$, respectively. Note that in this case both populations survive and approach an equilibrium. 


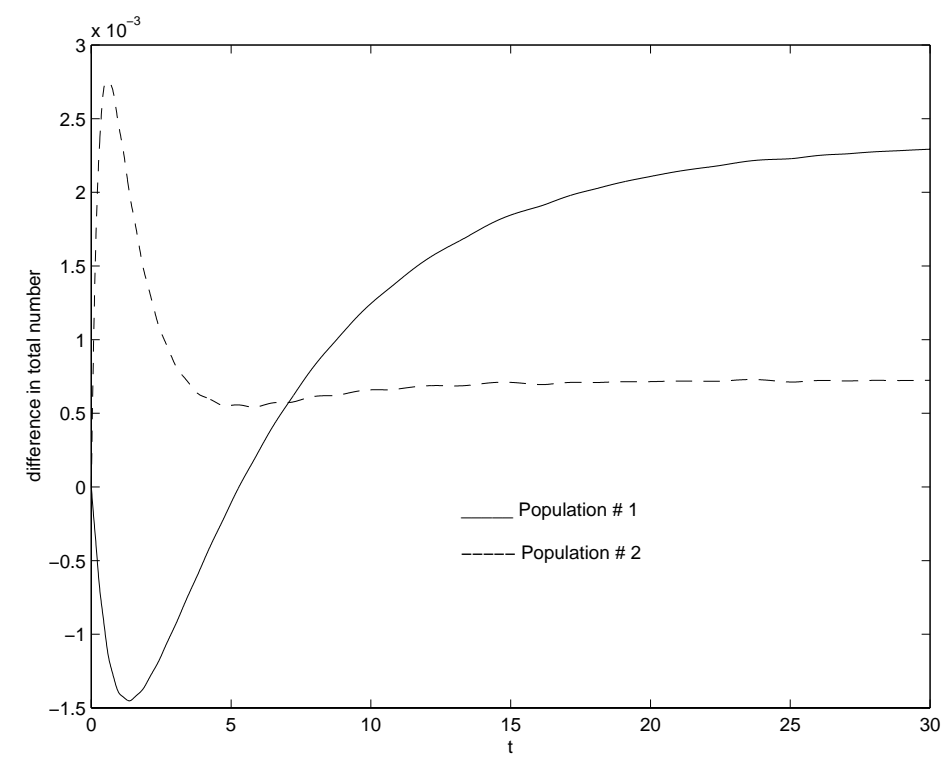

Figure 1: The difference between the total population resulting from the finite difference scheme and the total population resulting from solving the differential equations system using Runge-Kutta routine.

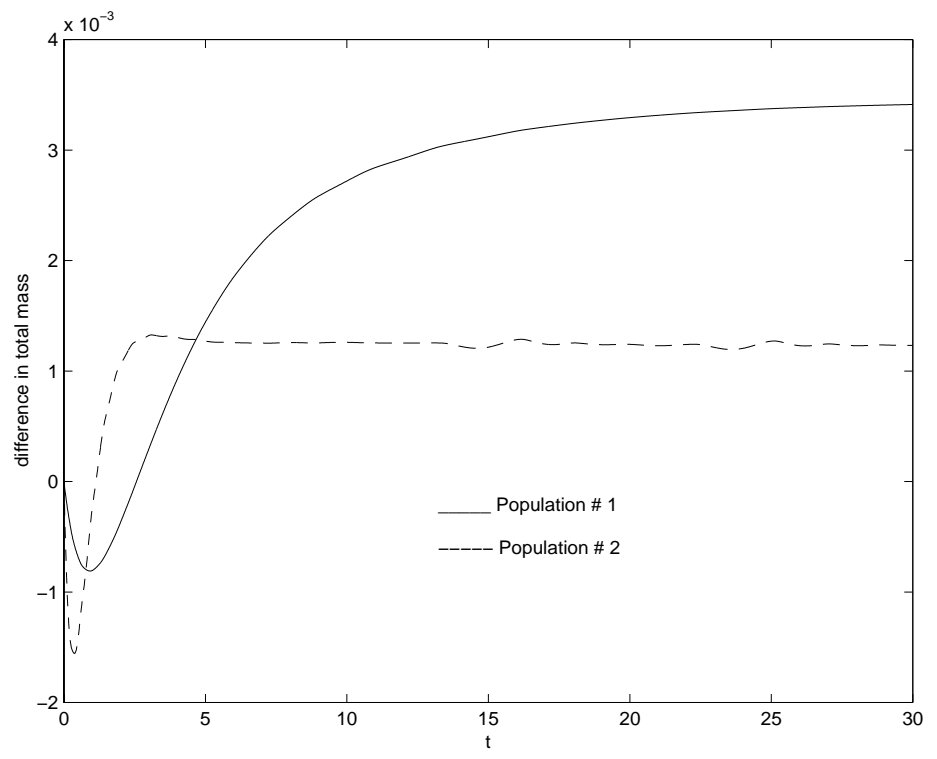

Figure 2: The difference between the total mass resulting from the finite difference scheme and the total mass resulting from solving the differential equations system using Runge-Kutta routine. 


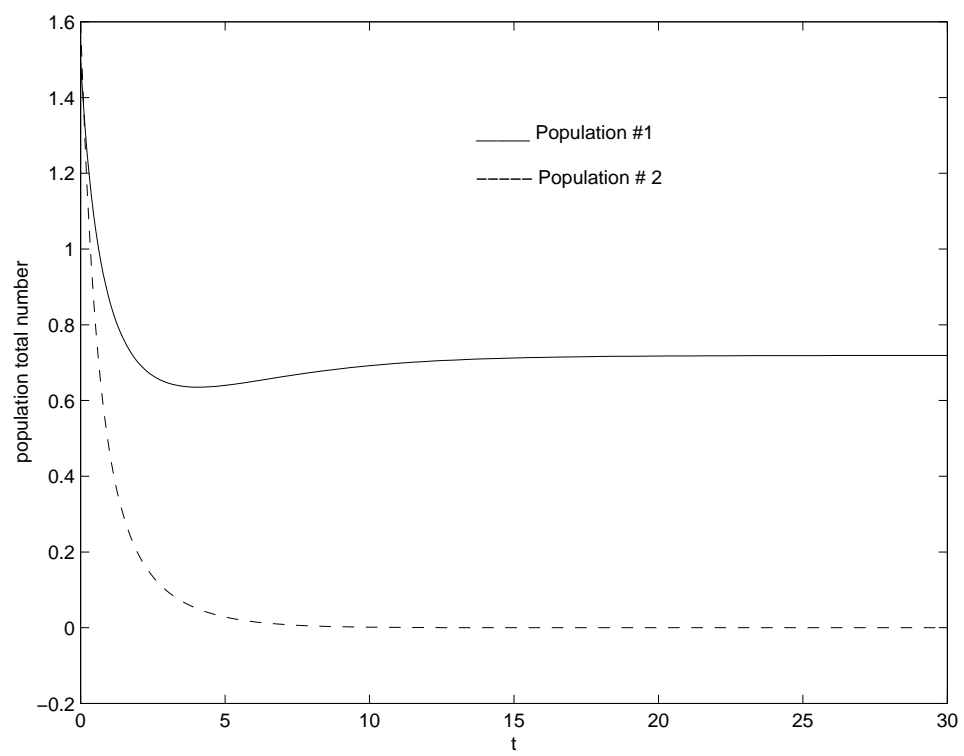

Figure 3: The computed total population $\left(P^{I}\right)^{\Delta x}(t), I=1,2$, on the interval $[0,30]$.

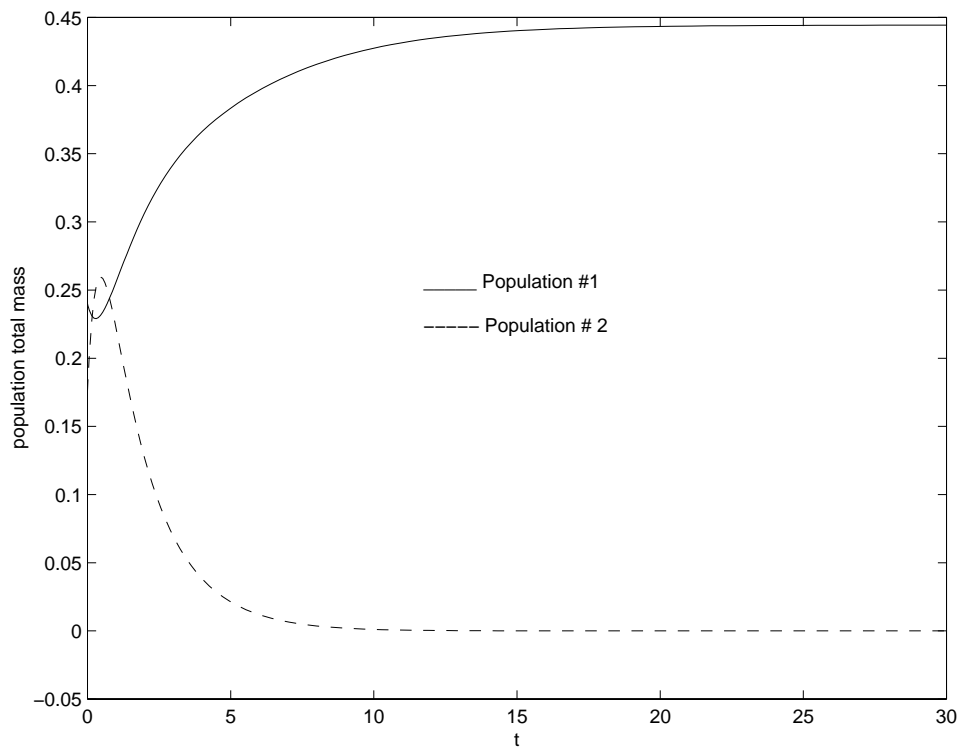

Figure 4: The computed total mass $\left(Q^{I}\right)^{\Delta x}(t), I=1,2$, on the interval $[0,30]$. 


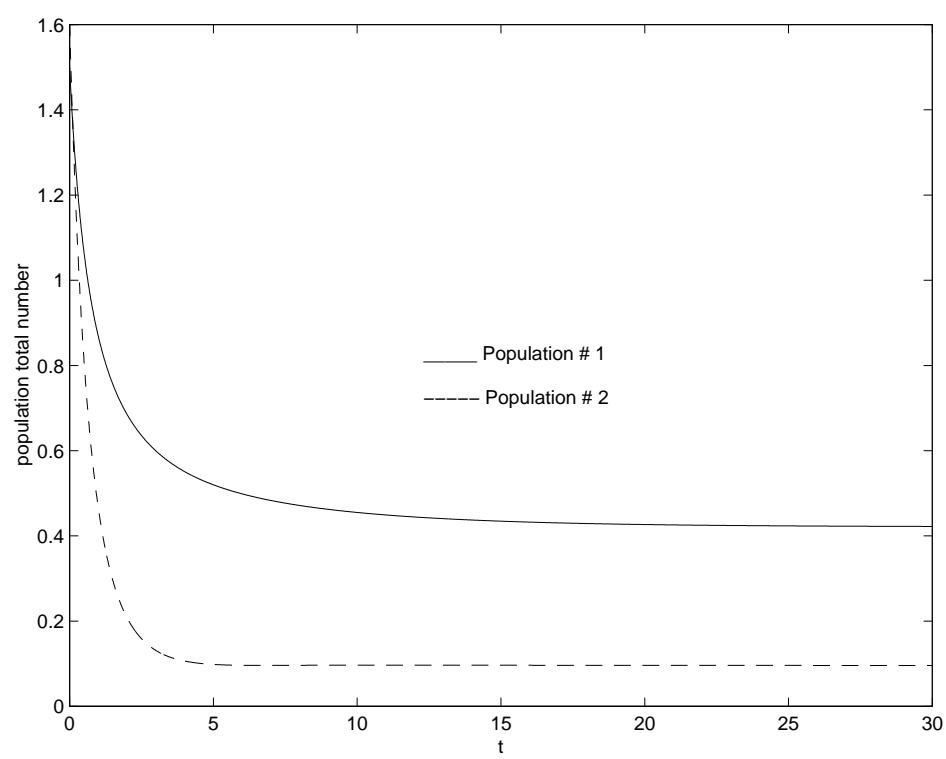

Figure 5: The computed total population $\left(P^{I}\right)^{\Delta x}(t), I=1,2$, on the interval $[0,30]$.

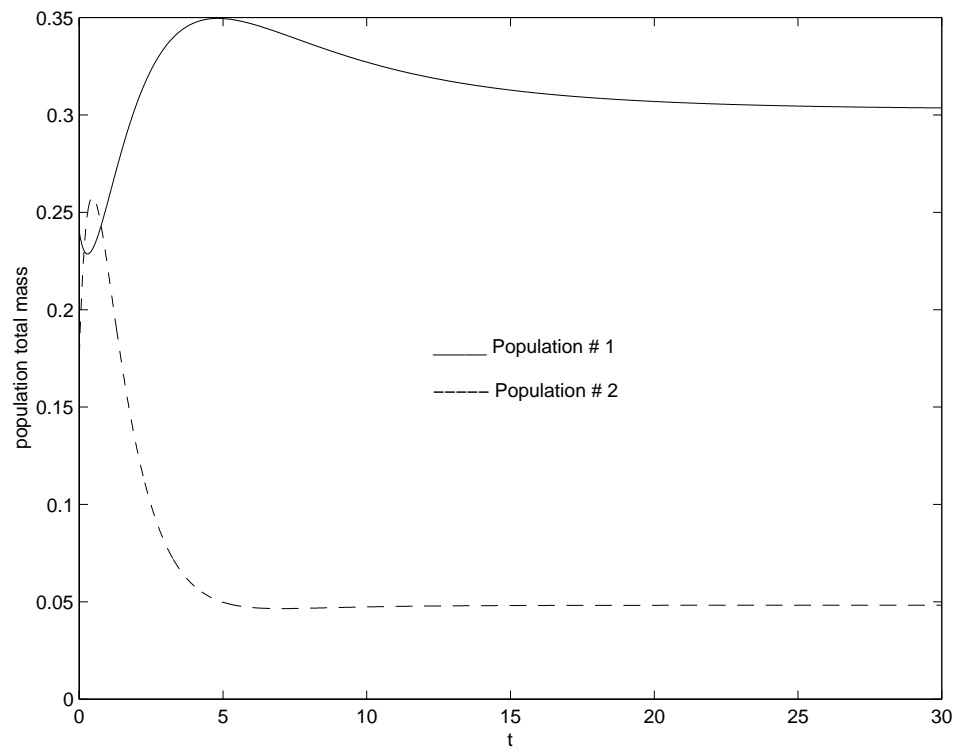

Figure 6: The computed total mass $\left(Q^{I}\right)^{\Delta x}(t), I=1,2$, on the interval $[0,30]$. 


\section{Regularity of Weak Solutions}

The goal of this section is to show that the bounded variation weak solution of problem (1) is continuous provided that the initial distributions $u^{I, 0}, I=1, \ldots, N$, satisfy the following compatibility conditions:

(H9) Let $P^{0}=\sum_{I=0}^{N} \int_{0}^{x_{\max }} w^{I}(x) u^{I, 0}(x) d x, g^{I, 0}(x)=g^{I}\left(x, P^{0}\right), \beta^{I, 0}(x)=\beta^{I}\left(x, P^{0}\right)$ and $m^{I, 0}(x)=m^{I}\left(x, P^{0}\right), I=1, \ldots, N$. Assume that the function $u^{I, 0}$ is a nonnegative continuous function and satisfies

$$
g^{I, 0}(0) u^{I, 0}(0)=C^{I}(0)+\sum_{J=1}^{N} \int_{0}^{x_{\max }} \gamma^{I, J} \beta^{J, 0}(x) u^{J, 0}(x) d x, \quad I=1, \ldots N
$$

It is worth pointing out that the following result demonstrates the remarkable difference between nonlocal quasilinear hyperbolic initial-boundary value problems similar to (1) and local quasilinear hyperbolic problems since it is well known that solutions of local problems can attain discontinuities in finite time.

Theorem 9 Under the additional assumption (H9) the bounded variation weak solution of problem (1) is continuous.

Proof. Let $\hat{\beta}^{I}$ and $\hat{m}^{I}$ be nonnegative continuously differentiable functions. Furthermore, assume that $\hat{g}^{I}$ is twice continuously differentiable in $x$ and continuously differentiable in $t, \hat{g}^{I}(x, t)>0, x \in\left[0, x_{\max }\right)$ and $\hat{g}^{I}\left(x_{\max }, t\right)=0, t \in[0, T]$. Consider the following initialboundary value problem:

$$
\left\{\begin{array}{l}
v_{t}^{I}+\left(\hat{g}^{I}(x, t) v^{I}\right)_{x}+\hat{m}^{I}(x, t) v^{I}=0, \quad(x, t) \in\left(0, x_{\max }\right) \times(0, T), \\
\hat{g}^{I}(0, t) v^{I}(0, t)=C^{I}(t)+\sum_{J=1}^{N} \int_{0}^{x_{\max }} \gamma^{I, J} \hat{\beta}^{J}(x, t) v^{J}(x, t) d x, \quad t \in(0, T), \\
v^{I}(x, 0)=u^{I, 0}(x), \quad x \in\left[0, x_{\max }\right] .
\end{array}\right.
$$


It follows from the results in Section 2 that problem (13) has a unique bounded variation weak solution. Furthermore, using standard arguments (e.g., see $[1,8,12,19]$ ) one can see that a characteristic curve passing through $(\widehat{x}, \widehat{t})$ is given by $\left(X^{I}(t ; \widehat{x}, \widehat{t}), t\right)$, where $X^{I}$ satisfies

$$
\frac{d}{d t} X^{I}(t ; \widehat{x}, \widehat{t})=\hat{g}^{I}(X(t ; \widehat{x}, \widehat{t}), t)
$$

and $X^{I}(\widehat{t} ; \widehat{x}, \widehat{t})=\widehat{x}$. By the assumptions on $\hat{g}^{I}$ the function $X^{I}$ is a strictly increasing function, and therefore a unique inverse function $\tau^{I}(x ; \widehat{x}, \widehat{t})$ exists. Hence if we define $G^{I}(x)=$ $\tau^{I}(x ; 0,0)$, then $\left(x, G^{I}(x)\right)$ represents the characteristic curve passing through $(0,0)$ and this curve divides the $(x, t)$-plane into two parts. And the weak solution $v^{I}$ of problem (13) has the following implicit representation

$$
v^{I}(x, t)= \begin{cases}u^{I, 0}\left(X^{I}(0 ; x, t)\right) \cdot & t \leq G^{I}(x), \\ \exp \left\{-\int_{0}^{t}\left[\hat{g}_{x}^{I}\left(X^{I}(s ; x, t), s\right)+m^{I}\left(X^{I}(s ; x, t), s\right)\right] d s\right\} & \\ R^{I}\left(\tau^{I}(0 ; x, t)\right) \cdot & t>G^{I}(x), \\ \exp \left\{-\int_{\tau^{I}(0 ; x, t)}^{t}\left[\hat{g}_{x}^{I}\left(X^{I}(s ; x, t), s\right)+\hat{m}^{I}\left(X^{I}(s ; x, t), s\right)\right] d s\right\} & \end{cases}
$$

where $R^{I}(t)=1 / \hat{g}^{I}(0, t)\left[C^{I}(t)+\sum_{J=1}^{N} \int_{0}^{x_{\max }} \gamma^{I, J} \hat{\beta}^{J}(x, t) v^{J}(x, t) d x\right]$.

Now, let $P(t)=\sum_{J=1}^{N} \int_{0}^{x_{\max }} w^{I}(x) u^{I}(x, t) d x$ where $u^{I}$ is the unique bounded variation weak solution of problem (1). Using (H2)-(H4) we see that $\hat{g}^{I}(x, t) \equiv g^{I}(x, P(t)), \hat{\beta}^{I}(x, t) \equiv$ $\beta^{I}(x, P(t))$ and $\hat{m}^{I}(x, t) \equiv m^{I}(x, P(t))$ satisfy the above requirements. Then, using (H8), the solution representation (14) and standard arguments as in [1] it follows that $v^{I}$ is continuous for this choice of parameters. Furthermore, by uniqueness of solutions, $v^{I}$ coincides with the solution component $u^{I}$ of the nonlinear problem $(1), I=1, \ldots, N$. This establishes the result 


\section{Concluding Remarks}

In this paper we presented a model that describes the evolution of $N$ subpopulations competing for common resources. Our numerical results indicate that the parameters $\gamma^{I, J}$ play a crucial role in the dynamics of these subpopulations. Several interesting questions about the model (1) arise naturally: What is a good measure (in terms of the rates $g^{I}$, $m^{I}$ and $\beta^{I}$ ) that will lead to the survival of the fittest in a closed reproduction case? In an open reproduction case, which populations will survive and which will go to extinction? We mention that for special cases of structured age and age-size population models, it was proved in [17] that under closed reproduction a good measure of species fitness is the product of the birth rate function and the survivorship function. To our knowledge, however, no results concerning the open reproduction case for structured populations are available. For a classical Lotka-Volterra competition model which is represented by a system of $N$ differential equations, conditions on the growth and mortality rates of each population that will result in its survival or extinction have been discussed recently by several researchers (e.g., see $[5,6,7,21,22])$. Our future efforts will focus on generalizing such results to the distributed rate structured model presented in (1).

\section{ACKNOWLEDGMENTS}

The research of the first author was supported in part by the Louisiana Education Quality Support Fund under grant LEQSF(1996-99)-RD-A-36, while the research of the second author was supported in part by the Air Force Office of Scientific Research under grant AFOSR F49620-98-1-0180. 


\section{References}

[1] A.S. Ackleh and K. Deng, A Monotone Approximation for the Nonautonomous SizeStructured Population Model, Quart. Appl. Math., 57 (1999), 261-267.

[2] A.S. Ackleh and K. Deng, A Monotone Approximation for a Nonlinear Nonautonomous Size-Structured Population Model, Appl. Math. Comput., 108 (2000), 103-113.

[3] A.S. Ackleh and K. Ito, An Implicit Finite Difference Scheme for the Nonlinear Size Structure Model, Numer. Funct. Anal. Optim., 18 (1997), 865-884.

[4] A.S. Ackleh, D. Marshall, B.G. Fitzpatrick and H.E. Heatherly, Survival of the Fittest in a Generalized Logistic Model, Math. Models Methods Appl. Sci., 9 (1999), 1379-1391.

[5] S. Ahmed, Extinction of Species in Nonautonomous Lotka-Volterra Systems, Proc. Amer. Math. Soc., 127 (1999), 2905-2910.

[6] S. Ahmed and A.C. Lazer, One Species Extinction in an Autonomous Competition Model, Proc. First World Congress Nonlinear Analysts, Walter DeGruyter, Berlin, 1995.

[7] S. Ahmed and F. Montes de Oca, Extinction in Nonautonomous T-periodic LotkaVolterra System, Appl. Math. Comput., 90 (1998), 155-166.

[8] H.T. Banks, L.W. Botsford, F. Kappel and C. Wang, Modeling and Estimation in Size Structured Population Models, Math. Ecology, T.G. Hallam, L.J. Gross and S.A. Levin (Eds.), Singapore: World Scientific, 1988, pp. 521-541.

[9] H.T. Banks and B.G. Fitzpatrick, Estimation of Growth Rate Distribution in Size Structure Population Models, Quart. Appl. Math., 49 (1991), 215-235.

[10] H.T. Banks, B.G. Fitzpatrick, L.K. Potter, and Y. Zhang, Estimation of Probability Distributions for Individual Parameters Using Aggregate Population Observations, Stochas- 
tic Analysis, Control, Optimization and Applications, W. Mceneaney, G. Yin, Q. Zhang (Eds.), Birkhäuser, 1998, pp. 353-371.

[11] H.T. Banks and F. Kappel, Transformation Semigroups and $L^{1}$-Approximation for Size Structure Population Models, Semigroup Forum, 38 (1989), 141-155.

[12] H.T. Banks, F. Kappel and C. Wang, Weak Solutions and Differentiability for Size Structured Population Models, Birkhäuser Internat. Ser. Numer. Math., 100 (1991), $35-50$.

[13] A. Calsina and J. Saldana, A Model of Physiologically Structured Population Dynamics with a Nonlinear Growth Rate, J. Math. Biol., 33 (1995), 335-364.

[14] M.G. Crandall and A. Majda, Monotone Difference Approximations for Scalar Conservation Laws, J. Math. Comp., 34 (1980), 1-21.

[15] B.G. Fitzpatrick, Vector Valued Measure Approach for a Size Structured Population Model, J. Math. Anal. Appl., 172 (1993), 73-91.

[16] B.G. Fitzpatrick, Rate Distribution Modeling for Structured Heterogeneous Populations, Birkhäuser Internat. Ser. Numer. Math., 118 (1994), 131-141.

[17] S.H. Henson and T.G. Hallam, Survival of the Fittest: Asymptotic Competitive Exclusion in Structured Population and Community Models, Nonlinear World, 1 (1994), $385-402$.

[18] W. Huyer, A Size Structured Population Model with Dispersion, J. Math. Anal. Appl., 181 (1994), 716-754.

[19] K. Ito, F. Kappel and G. Peichl, A Fully Discretized Approximation Scheme for SizeStructured Population Models., SIAM J. Numer. Anal., 28 (1991), 923-954. 
[20] R. Leveque, Numerical Methods for Conservation Laws, Birkhäuser, Boston, 1990.

[21] F. Montes de Oca and M.L. Zeeman, Balancing Survival and Extinction in Nonautonomous Competitive Lotka-Volterra Systems, J. Math. Anal. Appl., 192 (1995), 360370.

[22] F. Montes de Oca and M.L. Zeeman, Extinction in Nonautonomous Competitive LotkaVolterra Systems, Proc. Amer. Math. Soc., 124 (1996), 3677-3687.

[23] J. Smoller, Shock Waves and Reaction-Diffusion Equations, Springer-Verlag, New York, 1994. 